\title{
Shadowing in Inelastic Nucleon-Nucleon Cross Section?
}

\author{
Kari J. Eskola๑, ${ }^{\dagger}$ Ilkka Helenius $\odot,{ }^{*}$ Mikko Kuha $\odot,{ }^{*}$ and Hannu Paukkunen ${ }^{\S}$ \\ University of Jyvaskyla, Department of Physics, P.O. Box 35, FI-40014 University of Jyvaskyla, Finland \\ and Helsinki Institute of Physics, P.O. Box 64, FI-00014 University of Helsinki, Finland
}

(Received 2 April 2020; revised 9 July 2020; accepted 16 October 2020; published 18 November 2020)

\begin{abstract}
Experimental results of inclusive hard-process cross sections in heavy-ion collisions conventionally lean on a normalization computed from Glauber models where the inelastic nucleon-nucleon cross section $\sigma_{\mathrm{nn}}^{\text {inel }}$ - a crucial input parameter-is simply taken from proton-proton measurements. In this Letter, using the computed electroweak boson production cross sections in lead-lead collisions as a benchmark, we determine $\sigma_{\mathrm{nn}}^{\text {inel }}$ from the recent ATLAS data. We find a significantly suppressed $\sigma_{\mathrm{nn}}^{\text {inel }}$ relative to what is usually assumed, show the consequences for the centrality dependence of the cross sections, and address the phenomenon in an eikonal minijet model with nuclear shadowing.
\end{abstract}

DOI: 10.1103/PhysRevLett.125.212301

Introduction.-In high-energy nucleus-nucleus collisions, the main goals are to study the properties of strongly interacting matter and QCD dynamics in the nuclear environment (for a recent review see, e.g., [1]). In these collisions, the produced particle multiplicity correlates strongly with the collision geometry: the more central the collision, typically the higher the multiplicity. Experimentally, the centrality classification is obtained by ordering the events according to their multiplicity or transverse energy into bins of equal fraction, say $10 \%$, of all events. Conventionally, 0\%-10\% (90\%-100\%) centrality refers to the events of highest (lowest) multiplicities and $0 \%-100 \%$ to all events, minimum bias.

Inclusive hard processes ( $h$ for hard) in turn are rarer processes of a large momentum scale whose cross sections in nucleus-nucleus collisions are traditionally obtained by converting the measured per-event yields $N_{h}^{c} / N_{\mathrm{evt}}^{c}$ in a centrality class $c$ into hard nucleon-nucleon cross sections $\sigma_{h}^{c}$ through

$$
\sigma_{h}^{c}=\frac{\sigma_{\mathrm{nn}}^{\mathrm{inel}}}{\left\langle N_{\mathrm{bin}}\right\rangle_{c}} \frac{N_{h}^{c}}{N_{\mathrm{evt}}^{c}}
$$

where $\left\langle N_{\text {bin }}\right\rangle_{c}$ is the mean number of independent inelastically interacting nucleon-nucleon pairs, binary collisions, in the centrality class $c$, and $\sigma_{\mathrm{nn}}^{\mathrm{inel}}$ is the inelastic nucleonnucleon cross section. The model-dependent quantity $\left\langle N_{\text {bin }}\right\rangle_{c}$ here is obtained from the Monte Carlo (MC) Glauber model [2]. The nuclear modification ratio $R_{A A}^{h, c}$

Published by the American Physical Society under the terms of the Creative Commons Attribution 4.0 International license. Further distribution of this work must maintain attribution to the author(s) and the published article's title, journal citation, and DOI. Funded by SCOAP ${ }^{3}$. is then obtained by dividing $\sigma_{h}^{c}$ by the corresponding minimum-bias cross section in proton-proton collisions where $A$ refers to the colliding nuclei.

This method of constructing hard cross sections is a routine procedure in the heavy-ion measurements at RHIC and at the LHC, and it has been used, e.g., to construct nuclear modification ratios for jets [3-6] and hadrons [7-13], which, in turn, are widely used in theoretical studies of jet quenching [14-16] and partonic energy loss [17-20]. In the same way, Eq. (1) forms the basis for measuring centrality-dependent cross sections of direct photon [21-23] and electroweak (EW) boson [24-27] production, which can be used to study, e.g., nuclear effects in parton distribution functions (PDFs) [28,29].

The basic inputs of the Glauber model are the nuclear geometry and $\sigma_{\mathrm{nn}}^{\text {inel }}[2]$. In the MC Glauber model, the positions of the nucleons are sampled event by event according to the nuclear density profile, usually the Woods-Saxon distribution [30]. The probability for an interaction between two nucleons depends on their mutual distance and $\sigma_{\mathrm{nn}}^{\text {inel }}$. As a result, cross-section measurements through Eq. (1) depend on $\sigma_{\mathrm{nn}}^{\text {inel }}$ in a nontrivial way. An established procedure dating back to early fixed-target experiments, such as E178 at Fermilab [31], is to take the value of $\sigma_{\mathrm{nn}}^{\text {inel }}$ and its energy dependence from protonproton measurements. However, at high enough energies, like those at the LHC, the particle production becomes sensitive to QCD dynamics at small momentum fractions $x$ where some suppression is expected due to gluon shadowing [32-34] or saturation phenomena [35-37]. Such effects become more pronounced in heavy nuclei and toward lower scales, so one could argue that, in collisions involving heavy ions, the value of $\sigma_{\mathrm{nn}}^{\text {inel }}$ should also be reduced relative to what is measured in proton-proton collisions. Through Eq. (1), this would then change the obtained hard cross sections and nuclear modification ratios and thereby affect 
all the subsequent analyses that take these measured cross sections as an input. In this way, the value of $\sigma_{\mathrm{nn}}^{\text {inel }}$ is critical and could have far-reaching consequences, e.g., for the precision studies of jet quenching and other related phenomena. Thus, an alternative benchmark for $\sigma_{\mathrm{nn}}^{\text {inel }}$ is called for.

As proposed in Ref. [28], the Glauber model and its inputs could be tested by studying the production of well known "standard candles," such as EW bosons, in $\mathrm{Pb}+\mathrm{Pb}$ collisions at the LHC, but so far this has been limited by the precision of the LHC Run-I measurements [24-26,38]. Thanks to the increased luminosity and collision energy of Run II, the recent $W^{ \pm}$- and Z-boson measurements by ATLAS $[39,40]$ have pushed the precision to a few-percent level, enabling now a more precise Glauber model calibration. In the present Letter, we use these ATLAS data to study the possible nuclear suppression of $\sigma_{\mathrm{nn}}^{\text {inel }}$ in $\mathrm{Pb}+\mathrm{Pb}$ collisions. Since the ALICE measurement [41] is less precise and has no reference $p+p$ data, we leave it out of the analysis. The idea is to first establish the EW-boson cross sections by using next-to-next-to-leading order (NNLO) perturbative QCD (pQCD) with state-of-the-art PDFs for protons and nuclei. Using the theory prediction on the left-hand side of Eq. (1), we can then determine $\sigma_{\mathrm{nn}}^{\text {inel }}$ within the same MC Glauber implementation as in the experimental analyses. We find that the data favor a significant suppression in $\sigma_{\mathrm{nn}}^{\text {inel }}$. We also demonstrate that the unexpected enhancement seen by ATLAS in the ratios $R_{\mathrm{PbPb}}^{W^{ \pm}, Z}$ toward peripheral collisions disappears with the found smaller value of $\sigma_{\mathrm{nn}}^{\text {inel }}$. In addition, we show that the suppression of $\sigma_{\mathrm{nn}}^{\text {inel }}$ is compatible with predictions from an eikonal minijet model with nuclear shadowing.

Nuclear suppression in $\sigma_{\mathrm{nn}}^{\text {inel }}$.-The observables we exploit in this work to extract $\sigma_{\mathrm{nn}}^{\text {inel }}$ are the rapiditydependent nuclear modification ratios for $W^{ \pm}$and Z-boson production in different centrality classes. Experimentally these are defined as

$$
R_{\mathrm{PbPb}}^{\exp }(y)=\frac{1}{\left\langle T_{\mathrm{PbPb}}\right\rangle} \frac{\frac{1}{N_{\mathrm{evt}}} d N_{\mathrm{PbPb}}^{W^{ \pm}, Z} / d y}{d \sigma_{\mathrm{Pp}}^{W^{ \pm}, Z} / d y},
$$

where the per-event yield is normalized into nucleonnucleon cross section by diving with the mean nuclear overlap $\left\langle T_{\mathrm{PbPb}}\right\rangle=\left\langle N_{\text {bin }}\right\rangle_{c} / \sigma_{\mathrm{nn}}^{\text {inel }}$ obtained from a MC Glauber model calculation. For minimum-bias collisions, the same quantity can be calculated directly as a ratio between the cross sections in $\mathrm{Pb}+\mathrm{Pb}$ and $\mathrm{p}+\mathrm{p}$ collisions,

$$
R_{\mathrm{PbPb}}^{\text {theor }}(y)=\frac{1}{(208)^{2}} \frac{d \sigma_{\mathrm{PbPb}}^{W^{ \pm}, Z} / d y}{d \sigma_{\mathrm{pp}}^{W^{ \pm}, Z} / d y},
$$

which is completely independent of the Glauber modeling. We have calculated the cross sections in Eq. (3) at NNLO with the MCFM code (version 8.3) [42]. For the protons, we use the recent NNPDF3.1 PDFs [43], which provide an excellent agreement to ATLAS data for $W^{ \pm}$and Z-boson production in $\mathrm{p}+\mathrm{p}$ collisions at $\sqrt{s}=5.02 \mathrm{TeV}$ [44]. The nuclear modifications for the PDFs are obtained from the centrality-independent EPPS16 NLO analysis [45], which includes Run-I data for $W^{ \pm}$and $Z$ production in $\mathrm{p}+\mathrm{Pb}$ collisions at the LHC [46-48] and provides an excellent description of the more recent Run-II data [49]. The available NNLO nuclear PDFs [50,51] do not include any constraints beyond deeply inelastic scattering, so the applied PDFs provide currently the most accurately constrained setup for the considered observables. The factorization and renormalization scales are fixed to the respective EW-boson masses.

The ratios $R_{\mathrm{PbPb}}^{\mathrm{theor}}$ and $R_{\mathrm{PbPb}}^{\mathrm{exp}}$ are compared in the upper panel of Fig. 1. For $W^{ \pm}, R_{\mathrm{PbPb}}^{\mathrm{exp}}$ is formed by diving the normalized yield in $\mathrm{Pb}+\mathrm{Pb}$ from Ref. [39] with the corresponding cross section in $p+p$ from Ref. [44], adding the uncertainties in quadrature. The plotted experimental uncertainties do not include the uncertainty in $\left\langle T_{\mathrm{PbPb}}\right\rangle$. The theoretical uncertainties derive from the EPPS16 error sets

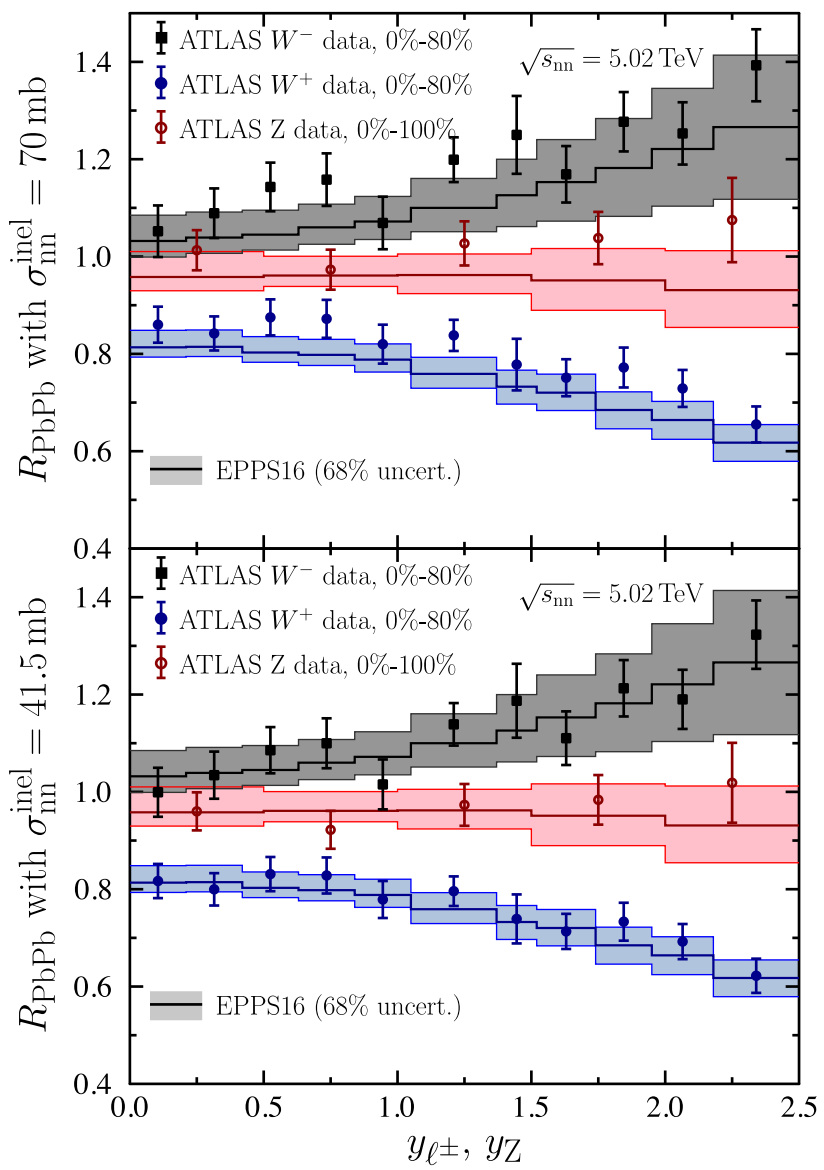

FIG. 1. Nuclear modification ratios of $W^{ \pm}$and $Z$, computed from $\mathrm{pQCD}$ (solid lines with error bands) and from ATLAS data $[39,40]$ with $\sigma_{\mathrm{nn}}^{\text {inel }}=70 \mathrm{mb}$ (upper panel) and $41.5 \mathrm{mb}$ (lower panel). 


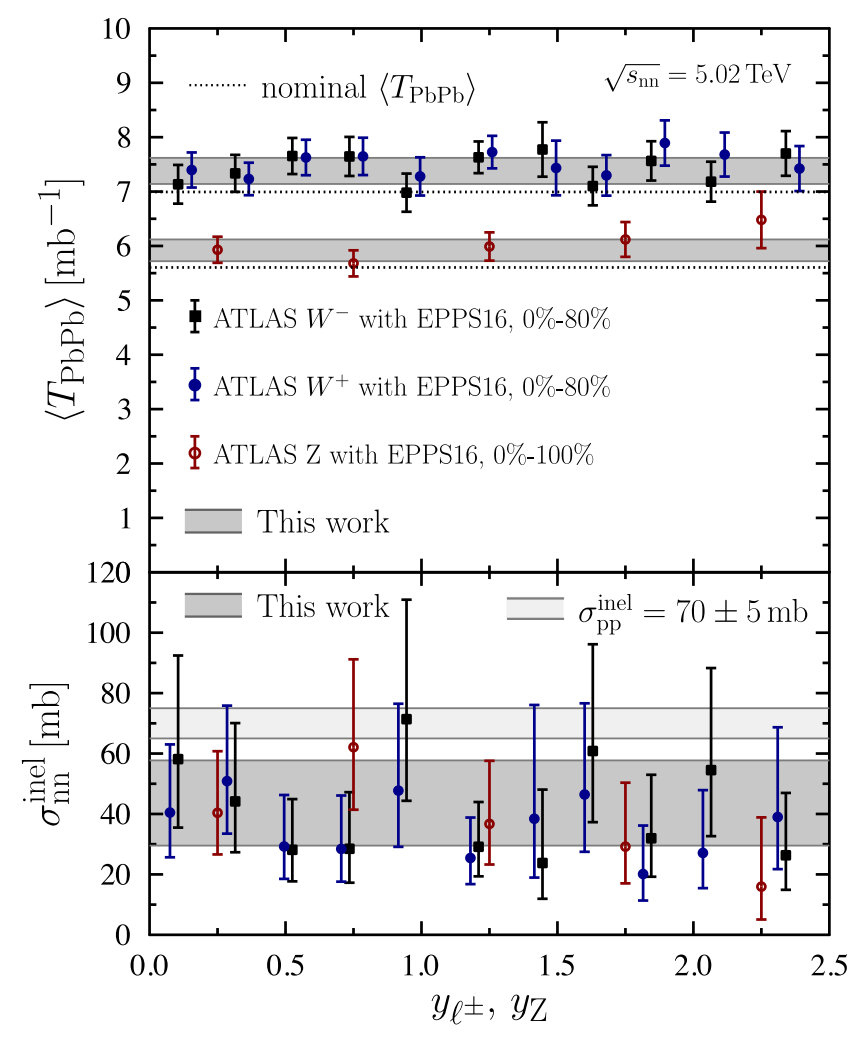

FIG. 2. Extracted values of the mean nuclear overlap functions (upper panel) and $\sigma_{\mathrm{nn}}^{\text {inel }}$ (lower panel). The dark gray bands show the values obtained by fitting $\sigma_{\mathrm{nn}}^{\text {inel }}$ and the dashed lines and the light gray band corresponds to the nominal $\sigma_{\mathrm{pp}}^{\mathrm{inel}}$.

and correspond to the $68 \%$ confidence level. Note that the $W^{ \pm}$measurement is for $0 \%-80 \%$ centrality instead of full 0\%-100\%. However, for rare processes like the EW bosons, the contribution from the $80 \%-100 \%$ region is negligible, so the comparison with the minimum-bias calculations is justified. It is evident that with $\sigma_{\mathrm{nn}}^{\text {inel }}=$ $70 \mathrm{mb}$ both the $W^{ \pm}$and the $Z$ data tend to lie above the calculated result, which we will interpret as an evidence of nuclear suppression in $\sigma_{\mathrm{nn}}^{\text {inel }}$ as explained below.

By equating Eqs. (2) and (3), we can convert each data point to $\left\langle T_{\mathrm{PbPb}}\right\rangle$. The outcome is shown in the upper panel of Fig. 2. The obtained values tend to be higher than the nominal $\left\langle T_{\mathrm{PbPb}}\right\rangle=5.605 \mathrm{mb}^{-1}(0 \%-100 \%)$ and $\left\langle T_{\mathrm{PbPb}}\right\rangle=$ $6.993 \mathrm{mb}^{-1}(0 \%-80 \%)$, which assume $\sigma_{\mathrm{nn}}^{\text {inel }}=70 \mathrm{mb}$ (see Table I). The fact that the preferred values of $\left\langle T_{\mathrm{PbPb}}\right\rangle$ are independent of the rapidity strongly suggests that the original mismatch in $R_{\mathrm{PbPb}}$ is a normalization issue-the nuclear PDFs predict the rapidity dependence correctly.

Since each $\left\langle T_{\mathrm{PbPb}}\right\rangle$ maps to $\sigma_{\mathrm{nn}}^{\text {inel }}$ through MC Glauber, we can also directly convert $R_{\mathrm{PbPb}}^{\exp }$ to $\sigma_{\mathrm{nn}}^{\text {inel }}$. Here, we have used TGlauberMC (version 2.4) [52], which is the same MC Glauber implementation as in the considered ATLAS analyses. The centrality classification is done with a two-component model, including negative binomial fluctuations [53] similar to the ALICE prescription [54] with parameters from Ref. [55]. The obtained values of $\left\langle T_{\mathrm{PbPb}}\right\rangle$ are in an excellent agreement with the ATLAS values in Refs. [39,40] in all centrality classes when using the nominal, unsuppressed value $\sigma_{\mathrm{nn}}^{\text {inel }}=70 \mathrm{mb}$. The values of $\sigma_{\mathrm{nn}}^{\text {inel }}$ extracted from each data point are shown in Fig. 2. It is obvious that the data prefer a value of $\sigma_{\mathrm{nn}}^{\text {inel }}$, which is less than the $\sigma_{\mathrm{pp}}^{\text {inel }}=70 \mathrm{mb}$ obtained from $\mathrm{p}+\mathrm{p}$ data.

To quantify the optimal $\sigma_{\mathrm{nn}}^{\text {inel }}$, we fit its value by requiring a match between $R_{\mathrm{PbPb}}^{\mathrm{exp}}$ and $R_{\mathrm{PbPb}}^{\mathrm{theor}}$ treating the EPPS16 uncertainties as Gaussian correlated errors. In practice, we define a $\chi^{2}$ function by

$$
\begin{aligned}
\chi^{2} & =\sum_{i}\left[\frac{\mathcal{N}_{i} R_{\mathrm{PbPb}, i}^{\mathrm{exp}}-R_{\mathrm{PbPb}, i}^{\mathrm{theer}}+\sum_{k} f_{k} \beta_{i}^{k}}{\mathcal{N}_{i} \delta_{i}^{\text {exp }}}\right]^{2}+T \sum_{k} f_{k}^{2} \\
\mathcal{N}_{i} & =\left\langle T_{\mathrm{PbPb}}^{i}\left(\sigma_{\mathrm{pp}}^{\text {inel }}\right)\right\rangle /\left\langle T_{\mathrm{PbPb}}^{i}\left(\sigma_{\mathrm{nn}}^{\text {inel }}\right)\right\rangle,
\end{aligned}
$$

where $i$ runs over the data points and $k=1, \ldots, 20$ over the number error-set pairs in EPPS16. The factors $\mathcal{N}_{i}$ with $\sigma_{\mathrm{pp}}^{\text {inel }}=70 \mathrm{mb}$ account for the shifted normalizations when $\sigma_{\mathrm{nn}}^{\text {inel }}$ changes. Also the data uncertainties $\delta_{i}^{\text {exp }}$ are scaled by this factor to avoid D'Agostini bias [56]. The tolerance $T=1.645^{2}$ in the penalty term takes into account scaling the $90 \%$ confidence limit uncertainties of EPPS16 into 68\% and $\beta_{i}^{k} \equiv\left[R_{\mathrm{PbPb}, i}^{\text {theor }}\left(S_{k}^{+}\right)-R_{\mathrm{PbPb}, i}^{\text {theor }}\left(S_{k}^{-}\right)\right] / 2$, where $S_{k}^{+}$and $S_{k}^{-}$ are the positive and negative variations, respectively, of EPPS 16 error sets. The $\chi^{2}$ is minimized with respect to $\sigma_{\mathrm{nn}}^{\text {inel }}$ and $f_{k}(1+20$ parameters $)$. We find

$$
\sigma_{\mathrm{nn}}^{\mathrm{inel}}=41.5_{-12.0}^{+16.2} \mathrm{mb},
$$

where the uncertainties follow from the $\Delta \chi^{2}=1$ criterion. The resulting values for $\left\langle T_{\mathrm{PbPb}}\right\rangle$ and $\sigma_{\mathrm{nn}}^{\text {inel }}$ are compared to the data-extracted values in Fig. 2, and the renormalized data for $R_{\mathrm{PbPb}}$ are compared to theoretical predictions in the lower panel of Fig. 1. It is worth stressing that different final states prefer a very similar suppressed value of $\sigma_{\mathrm{nn}}^{\text {inel }}$ and that a very good agreement in $R_{\mathrm{PbPb}}$ is found when normalizing with $\left\langle T_{\mathrm{PbPb}}\right\rangle$ calculated using the suppressed cross section in the MC Glauber calculation.

Centrality dependence.-Even the quite significant suppression in $\sigma_{\mathrm{nn}}^{\text {inel }}$ leads to rather modest modifications in $\left\langle T_{\mathrm{PbPb}}\right\rangle$ for central and (close-to) minimum-bias collisions. The impact, however, grows toward more peripheral centrality classes (see Table I). To illustrate this, Fig. 3 compares the centrality-dependent $R_{\mathrm{PbPb}}^{\exp }$ before and after rescaling the data by $\left\langle T_{\mathrm{PbPb}}\left(\sigma_{\mathrm{pp}}^{\text {inel }}\right)\right\rangle /\left\langle T_{\mathrm{PbPb}}\left(\sigma_{\mathrm{nn}}^{\text {inel }}\right)\right\rangle$ using the fitted $\sigma_{\mathrm{nn}}^{\text {inel }}$. The left-hand panels show the original ATLAS data, including the quoted $\left\langle T_{\mathrm{PbPb}}\right\rangle$ uncertainties. In the right-hand panels, the data have been rescaled, and the uncertainties follow from the $\sigma_{\mathrm{nn}}^{\text {inel }}$ fit. The striking effect is that the mysterious rise toward more peripheral collisions in the original data becomes compatible with a negligible centrality dependence, the central values indicating perhaps a mildly decreasing trend toward peripheral bins. As 
TABLE I. Mean nuclear overlap functions $\left\langle T_{\mathrm{PbPb}}\right\rangle[1 / \mathrm{mb}]$ for ATLAS centrality classes with nominal and fitted $\sigma_{\mathrm{nn}}^{\text {inel }}$.

\begin{tabular}{lcccc}
\hline \hline$\sigma_{\mathrm{nn}}^{\text {inel }}$ & $70.0 \mathrm{mb}$ & $57.7 \mathrm{mb}$ & $41.5 \mathrm{mb}$ & $29.5 \mathrm{mb}$ \\
\hline $0 \%-2 \%$ & 28.26 & 28.39 & 28.55 & 28.69 \\
$2 \%-4 \%$ & 25.51 & 25.67 & 25.91 & 26.10 \\
$4 \%-6 \%$ & 23.09 & 23.28 & 23.55 & 23.80 \\
$6 \%-8 \%$ & 20.94 & 21.14 & 21.45 & 21.73 \\
$8 \%-10 \%$ & 19.00 & 19.23 & 19.56 & 19.86 \\
$10 \%-15 \%$ & 16.08 & 16.31 & 16.67 & 17.02 \\
$15 \%-20 \%$ & 12.58 & 12.83 & 13.22 & 13.59 \\
$20 \%-25 \%$ & 9.762 & 10.01 & 10.40 & 10.78 \\
$25 \%-30 \%$ & 7.487 & 7.722 & 8.102 & 8.469 \\
$30 \%-40 \%$ & 4.933 & 5.138 & 5.474 & 5.808 \\
$40 \%-50 \%$ & 2.628 & 2.780 & 3.036 & 3.300 \\
$50 \%-60 \%$ & 1.281 & 1.378 & 1.550 & 1.733 \\
$60 \%-80 \%$ & 0.395 & 0.435 & 0.510 & 0.595 \\
$80 \%-100 \%$ & 0.052 & 0.060 & 0.076 & 0.096 \\
$0 \%-80 \%$ & 6.993 & 7.143 & 7.385 & 7.624 \\
$0 \%-100 \%$ & 5.605 & 5.726 & 5.923 & 6.118 \\
\hline \hline
\end{tabular}

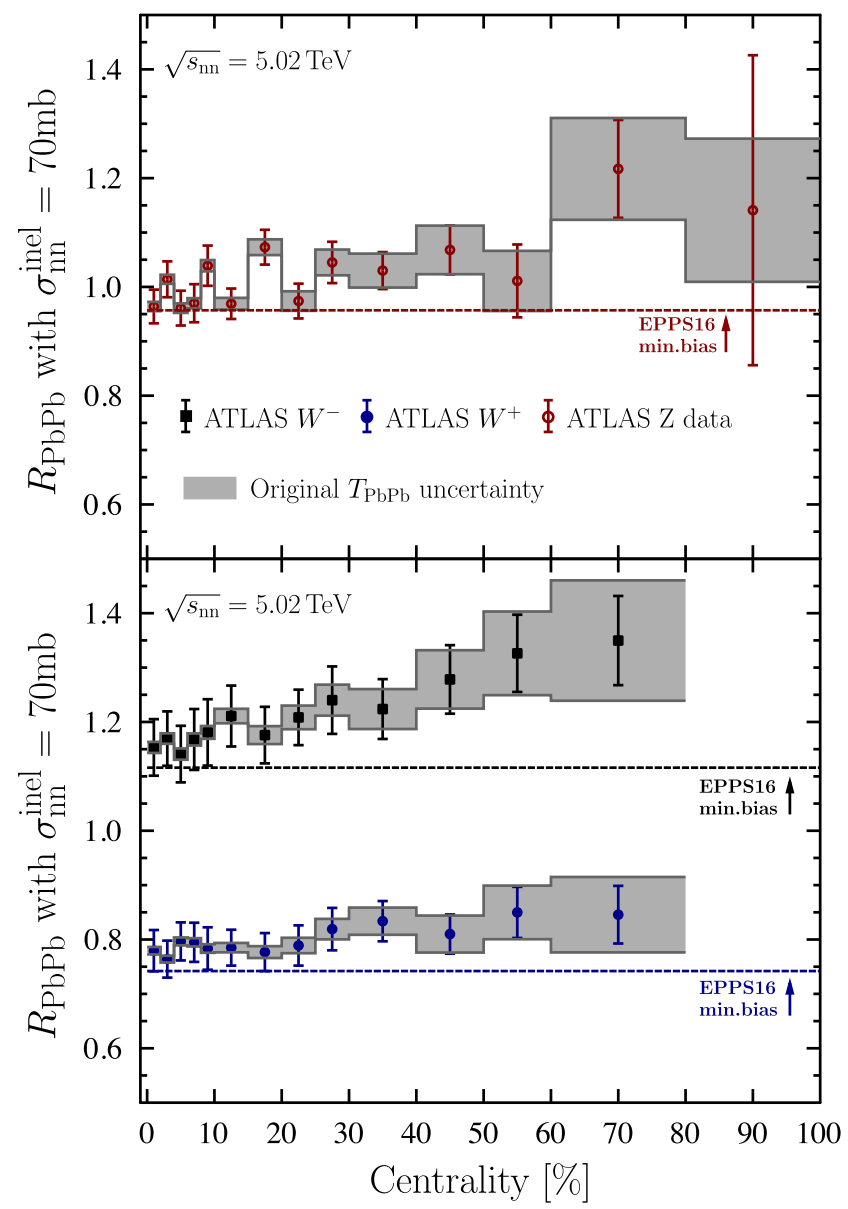

discussed, e.g., in the ATLAS publications $[39,40]$, such a suppression could be expected from selection and geometrical biases associated with the MC Glauber modeling [57]. Also other effects such as the possible centrality dependence of $\sigma_{\mathrm{nn}}^{\text {inel }}$ and the neutron-skin effect [58,59] may become relevant to explain the data behavior in the far periphery. However, for minimum-bias collisions, the suppression effect arises mainly from midcentral collisions $(0 \%-60 \%)$, so the resulting value for $\sigma_{\mathrm{nn}}^{\text {inel }}$ is robust against the phenomena occurring in the periphery.

Minijets with shadowing.-To study the plausibility of the obtained suppression in $\sigma_{\mathrm{nn}}^{\text {inel }}$, we calculate its value in an eikonal model for minijet production with nuclear shadowing. The model is based on a similar setup as in Ref. [60], but in the eikonal function we include only the contribution from the hard minijet cross section $\sigma_{\text {jet }}\left(\sqrt{s_{\mathrm{nn}}}, p_{0},[Q]\right)$, calculated at leading order in pQCD. The transversemomentum cutoff $p_{0}$ (which depends on $\sqrt{s_{\mathrm{nn}}}$, scale choice $Q$, and the proton thickness) and the width of the assumed Gaussian proton thickness function we fix so that the model reproduces $\sigma_{\mathrm{pp}}^{\text {inel }}=70 \mathrm{mb}$ matching the COMPETE

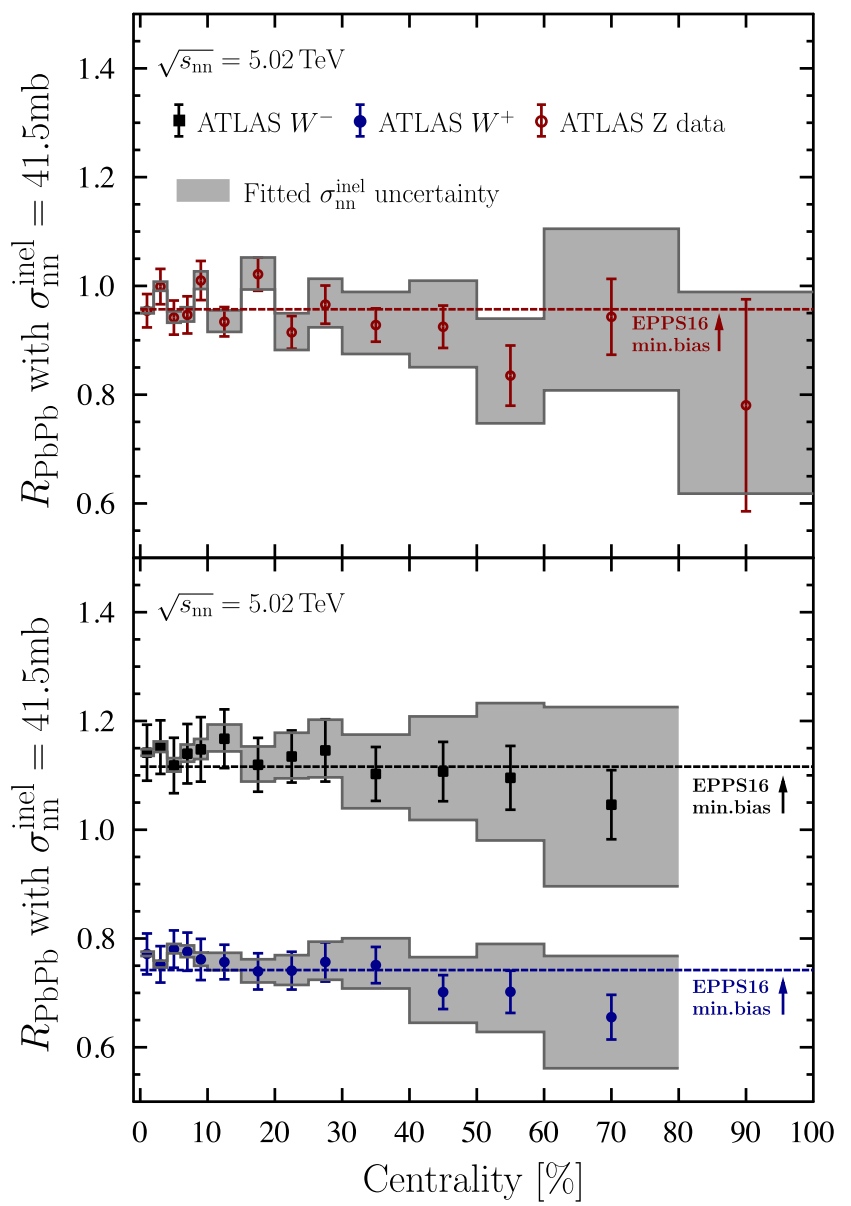

FIG. 3. The centrality-dependent nuclear modification ratios for $W^{ \pm}$and $Z$-boson production in $\mathrm{Pb}+\mathrm{Pb}$ collisions from ATLAS $[39,40]$ compared to NNLO pQCD calculation with EPPS16 nuclear modification with the nominal value of $\sigma_{\mathrm{nn}}^{\text {inel }}=70.0 \mathrm{mb}($ left $)$ and with the nuclear-suppressed value $\sigma_{\mathrm{nn}}^{\text {inel }}=41.5 \mathrm{mb}$ (right). 


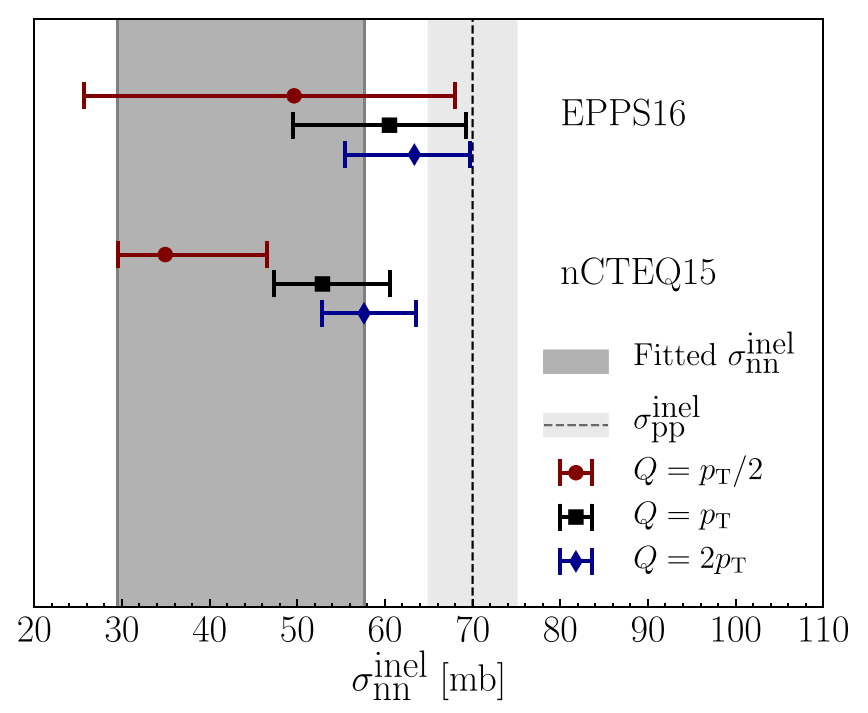

FIG. 4. Predictions for $\sigma_{\mathrm{nn}}^{\text {inel }}$ from an eikonal minijet model with the EPPS16 and nCTEQ15 nuclear PDFs for three scale choices. The fitted $\sigma_{\mathrm{nn}}^{\text {inel }}$ is indicated with the dark gray band and the nominal $\sigma_{\mathrm{pp}}^{\text {inel }}$ with the light gray band.

analysis [61] at $\sqrt{s}=5.02 \mathrm{GeV}$. The free proton PDFs are here CT14lo [62], and we take the nuclear PDF modifications from the EPPS16 [45] and nCTEQ15 [63] analyses. The results for $\sigma_{\mathrm{nn}}^{\text {inel }}$, obtained with $p_{0}$ and proton thickness function width fixed to the $\mathrm{p}+\mathrm{p}$ case, are shown in Fig. 4. The error bars are again from the nuclear PDFs scaled to the $68 \%$ confidence level. As expected at the few-GeV scales, the predicted $\sigma_{\mathrm{nn}}^{\text {inel }}$ depends strongly on the factorization and renormalization scale $Q$, but within the uncertainties the nuclear suppression obtained from the fits to the ATLAS $W^{ \pm}$and $Z$ data seems compatible with the eikonal model predictions with both nuclear PDFs.

Summary.-In the canonical approach, the normalization for the measured per-event yields in nuclear collisions is obtained from the Glauber model taking the value of $\sigma_{\mathrm{nn}}^{\text {inel }}$ from proton-proton measurements. Contrary to this, our strategy was to compare the state-of-the-art pQCD calculations with the measured $W^{ \pm}$and $Z$-boson $R_{\mathrm{PbPb}}$ and thereby unfold the value for $\sigma_{\mathrm{nn}}^{\text {inel }}$ at $\sqrt{s_{\mathrm{nn}}}=5.02 \mathrm{TeV}$. We find that the recent high-precision ATLAS data from Run II prefer the value $\sigma_{\mathrm{nn}}^{\text {inel }}=41.5_{-12.0}^{+16.2} \mathrm{mb}$, which is significantly lower than $\sigma_{\mathrm{pp}}^{\text {inel }}=70 \pm 5 \mathrm{mb}$. This new benchmark value for $\sigma_{\mathrm{nn}}^{\text {inel }}$ in $\sqrt{s_{\mathrm{nn}}}=5.02 \mathrm{TeV} \mathrm{Pb}+\mathrm{Pb}$ collisions is the main result of the present work. Such a suppression is in line with the expectations from an eikonal minijet model, including nuclear shadowing, but is not necessarily tied only to the shadowing phenomenon. Remarkably, when using the fitted value for $\sigma_{\mathrm{nn}}^{\text {inel }}$, the unexpected enhancements of $R_{\mathrm{PbPb}}$ in peripheral collisions disappear and the results become compatible with no centrality dependence. A possible hint of a slight decreasing trend toward peripheral collisions is observed that would be qualitatively in line with possible selection and geometrical biases. Our results thus suggest that the standard paradigm of using $\sigma_{\mathrm{pp}}^{\text {inel }}$ as an input to Glauber modeling potentially leads to a misinterpretation of the experimental data. The possible suppression could be further scrutinized by repeating the experimental analysis using a measured luminosity to convert the yields into cross sections or by measuring the total hadronic $\mathrm{Pb}+\mathrm{Pb}$ cross section at $\sqrt{s_{\mathrm{nn}}}=$ $5.02 \mathrm{GeV}$ with high enough precision.

We thank Mirta Dumancic for discussion concerning the ATLAS measurements. We acknowledge the Academy of Finland, projects 297058, 330448 (K. J. E.), 308301 (H. P. and I. H.), and 331545 (I. H.), and the Väisälä Foundation (M. K.) for financial support. The Finnish IT Center for Science (CSC) is acknowledged for the computing time through the Project No. jyy2580.

*Corresponding author.

ilkka.m.helenius@jyu.fi

†kari.eskola@jyu.fi

*mikko.a.kuha@student.jyu.fi

§hannu.t.paukkunen@jyu.fi

[1] W. Busza, K. Rajagopal, and W. van der Schee, Annu. Rev. Nucl. Part. Sci. 68, 339 (2018).

[2] M. L. Miller, K. Reygers, S. J. Sanders, and P. Steinberg, Annu. Rev. Nucl. Part. Sci. 57, 205 (2007).

[3] B. Abelev et al. (ALICE Collaboration), J. High Energy Phys. 03 (2014) 013.

[4] V. Khachatryan et al. (CMS Collaboration), Phys. Rev. C 96, 015202 (2017).

[5] G. Aad et al. (ATLAS Collaboration), Phys. Lett. B 719, 220 (2013).

[6] M. Aaboud et al. (ATLAS Collaboration), Phys. Lett. B 790, 108 (2019).

[7] A. M. Sirunyan et al. (CMS Collaboration), J. High Energy Phys. 10 (2018) 138.

[8] V. Khachatryan et al. (CMS Collaboration), J. High Energy Phys. 04 (2017) 039.

[9] G. Aad et al. (ATLAS Collaboration), J. High Energy Phys. 09 (2015) 050.

[10] S. Acharya et al. (ALICE Collaboration), Phys. Lett. B 788, 166 (2019).

[11] S. Acharya et al. (ALICE Collaboration), J. High Energy Phys. 11 (2018) 013.

[12] A. Adare et al. (PHENIX Collaboration), Phys. Rev. C 93, 024901 (2016).

[13] S. S. Adler et al. (PHENIX Collaboration), Phys. Rev. C 69, 034910 (2004).

[14] J. D. Bjorken, Report No. FERMILAB-PUB-82-059-THY (1982).

[15] G.-Y. Qin and X.-N. Wang, Int. J. Mod. Phys. E 24, 1530014 (2015); Quark-Gluon Plasma 5, edited by X.-N. Wang, (World Scientific, 2016), pp. 309-372, https://doi .org/10.1142/9789814663717_0007.

[16] D. d'Enterria, Relativistic Heavy Ion Physics, edited by R. Stock (Landolt-Börnstein, 2010), Vol. 23, Chap. 6.4, https:// doi.org/10.1007/978-3-642-01539-7_16. 
[17] F. Arleo, Phys. Rev. Lett. 119, 062302 (2017).

[18] C. Andrés, N. Armesto, M. Luzum, C. A. Salgado, and P. Zurita, Eur. Phys. J. C 76, 475 (2016).

[19] Y.-T. Chien, A. Emerman, Z.-B. Kang, G. Ovanesyan, and I. Vitev, Phys. Rev. D 93, 074030 (2016).

[20] K. C. Zapp, F. Krauss, and U. A. Wiedemann, J. High Energy Phys. 03 (2013) 080.

[21] G. Aad et al. (ATLAS Collaboration), Phys. Rev. C 93, 034914 (2016).

[22] S. Chatrchyan et al. (CMS Collaboration), Phys. Lett. B 710, 256 (2012).

[23] S. Afanasiev et al. (PHENIX Collaboration), Phys. Rev. Lett. 109, 152302 (2012).

[24] G. Aad et al. (ATLAS Collaboration), Eur. Phys. J. C 75, 23 (2015).

[25] G. Aad et al. (ATLAS Collaboration), Phys. Rev. Lett. 110, 022301 (2013).

[26] S. Chatrchyan et al. (CMS Collaboration), Phys. Lett. B 715, 66 (2012).

[27] S. Chatrchyan et al. (CMS Collaboration), Phys. Rev. Lett. 106, 212301 (2011).

[28] H. Paukkunen and C. A. Salgado, J. High Energy Phys. 03 (2011) 071.

[29] I. Helenius, K. J. Eskola, and H. Paukkunen, J. High Energy Phys. 05 (2013) 030.

[30] R. D. Woods and D. S. Saxon, Phys. Rev. 95, 577 (1954).

[31] J. E. Elias, W. Busza, C. Halliwell, D. Luckey, P. Swartz, L. Votta, and C. Young, Phys. Rev. D 22, 13 (1980).

[32] J.-w. Qiu, Nucl. Phys. B291, 746 (1987).

[33] N. Armesto, J. Phys. G 32, R367 (2006).

[34] L. Frankfurt, V. Guzey, and M. Strikman, Phys. Rep. 512, 255 (2012).

[35] L. V. Gribov, E. M. Levin, and M. G. Ryskin, Phys. Rep. 100, 1 (1983).

[36] L. D. McLerran and R. Venugopalan, Phys. Rev. D 49, 2233 (1994).

[37] F. Gelis, E. Iancu, J. Jalilian-Marian, and R. Venugopalan, Annu. Rev. Nucl. Part. Sci. 60, 463 (2010).

[38] S. Chatrchyan et al. (CMS Collaboration), J. High Energy Phys. 03 (2015) 022.

[39] G. Aad et al. (ATLAS Collaboration), Eur. Phys. J. C 79, 935 (2019).

[40] G. Aad et al. (ATLAS Collaboration), Phys. Lett. B 802, 135262 (2020).

[41] S. Acharya et al. (ALICE Collaboration), Phys. Lett. B 780, 372 (2018).

[42] R. Boughezal, J. M. Campbell, R. K. Ellis, C. Focke, W. Giele, X. Liu, F. Petriello, and C. Williams, Eur. Phys. J. C 77, 7 (2017).
[43] R. D. Ball et al. (NNPDF Collaboration), Eur. Phys. J. C 77, 663 (2017).

[44] M. Aaboud et al. (ATLAS Collaboration), Eur. Phys. J. C 79, 128 (2019); 79, 374(E) (2019).

[45] K. J. Eskola, P. Paakkinen, H. Paukkunen, and C. A. Salgado, Eur. Phys. J. C 77, 163 (2017).

[46] V. Khachatryan et al. (CMS Collaboration), Phys. Lett. B 750, 565 (2015).

[47] V. Khachatryan et al. (CMS Collaboration), Phys. Lett. B 759, 36 (2016).

[48] G. Aad et al. (ATLAS Collaboration), Phys. Rev. C 92, 044915 (2015).

[49] A. M. Sirunyan et al. (CMS Collaboration), Phys. Lett. B 800, 135048 (2020).

[50] M. Walt, I. Helenius, and W. Vogelsang, Phys. Rev. D 100, 096015 (2019).

[51] R. A. Khalek, J. J. Ethier, and J. Rojo (NNPDF Collaboration), Eur. Phys. J. C 79, 471 (2019).

[52] C. Loizides, J. Nagle, and P. Steinberg, SoftwareX 1-2, 13 (2015).

[53] While modifying $\sigma_{\mathrm{nn}}^{\text {inel }}$, the parameters of the two-component model should be adjusted to maintain a good description of the measured multiplicity or transverse energy distribution. However, as the change in $\sigma_{\mathrm{nn}}^{\text {inel }}$ can be accurately compensated purely by increasing the mean of the negative binomial distribution not affecting the resulting $\left\langle T_{\mathrm{PbPb}}\right\rangle$, the presented results would remain unmodified. Similarly, the observed increase of $T_{\mathrm{PbPb}}$ is not sensitive to the applied centrality selection criterion.

[54] B. Abelev et al. (ALICE Collaboration), Phys. Rev. C 88, 044909 (2013).

[55] ALICE Collaboration, Report No. ALICE-PUBLIC-2018011 (2018).

[56] G. D’Agostini, Nucl. Instrum. Methods Phys. Res., Sect. A 346, 306 (1994).

[57] C. Loizides and A. Morsch, Phys. Lett. B 773, 408 (2017).

[58] H. Paukkunen, Phys. Lett. B 745, 73 (2015).

[59] I. Helenius, H. Paukkunen, and K. J. Eskola, Eur. Phys. J. C 77, 148 (2017).

[60] X.-N. Wang, Phys. Rev. D 43, 104 (1991).

[61] J. R. Cudell, V. V. Ezhela, P. Gauron, K. Kang, Yu. V. Kuyanov, S. B. Lugovsky, E. Martynov, B. Nicolescu, E. A. Razuvaev, and N. P. Tkachenko (COMPETE Collaboration), Phys. Rev. Lett. 89, 201801 (2002).

[62] S. Dulat, T.-J. Hou, J. Gao, M. Guzzi, J. Huston, P. Nadolsky, J. Pumplin, C. Schmidt, D. Stump, and C.P. Yuan, Phys. Rev. D 93, 033006 (2016).

[63] K. Kovarik et al., Phys. Rev. D 93, 085037 (2016). 